\title{
Modelos Animais Alternativos para Estimação de Componentes de (co)Variância e de Parâmetros Genéticos e Fenotípicos do Peso ao Nascer na Raça Nelore ${ }^{1}$
}

\author{
Márcia Tereza Vieira Scarpati ${ }^{2}$, Raysildo Barbosa Lôbo ${ }^{3}$
}

\begin{abstract}
RESUMO - O objetivo deste trabalho foi estudar os aspectos genético-quantitativos do peso ao nascer (PN), de animais da raça Nelore, em rebanhos participantes do Programa de Melhoramento Genético da Raça Nelore, realizado pelo Departamento de Genética da Universidade de São Paulo. Apenas os pesos de bezerros normais, nascidos vivos e oriundos de gestações não gemelares, concebidos por meio da inseminação artificial foram considerados. Os componentes de (co)variância foram estimados assumindo-se quatro diferentes modelos lineares mistos, em que se atribuíram como causas de variação os efeitos genéticos direto e materno e de ambiente permanente, pela inclusão alternada dos efeitos aleatórios pertinentes, além dos efeitos fixos de grupo contemporâneo e classe de idade da vaca ao parto, nos modelos de análise. Este procedimento permitiu quantificar a importância das diferentes formas de influência na característica, especialmente as de origem materna. O número de observações foi 6511 ; a média, $31 \mathrm{~kg}$; e o desvio-padrão, 4,1 kg. As estimativas de herdabilidades direta, materna e total foram de 0,20 a 0,$37 ; 0,11$ a 0,13 ; e 0,22 a 0,37, respectivamente. As correlações entre efeitos direto e materno foram negativas $(-0,14$ a -0,16). O PN é uma característica predominantemente determinada por ação gênica aditiva direta, pouco influenciada pelo fenótipo materno, e com antagonismo entre os efeitos genéticos. A resposta à seleção para o PN poderá ser maximizada se baseada em estimativas obtidas em análises realizadas com modelos animais, em que os efeitos genéticos e maternos tenham sido considerados.
\end{abstract}

Palavras-chave: bovinos de corte, efeitos maternos, máxima verossimilhança restrita, seleção

\section{Alternative Animal Models to Estimate (co) Variance Components and Genetic and Phenotypic Parameters for Birth Weight in Nellore Cattle}

\begin{abstract}
The objective of this work was to study the genetic-quantitative aspects of birth weight (BW), in animals of Nellore breed, in the herd from the Nellore Cattle Genetic Improvement Program, conducted by the Department of Genetics of the University of São Paulo. Only the weights of sound calves, born alive and from births, obtained by artificial insemination were considered. The (co) variance components were estimated under four different animal models where direct and maternal genetic and permanent environmental were taken into account by including the appropriate random effects, as well as contemporary groups and age of cow at calving effects, in the statistical model. This procedure allowed the quantification of the contribution of different random effects, especially maternal effects. The number of records was 6511 ; the overall means $31 \mathrm{~kg}$, and the standard deviation $4,1 \mathrm{~kg}$. The direct, maternal and total estimated heritability ranged from 0.20 to $0.37,0.11$ to 0.13 and 0.22 to 0.37 , respectively. The correlation's between genetic direct and maternal effects were negative $(-0,14$ to $-0,16)$. The BW is a trait greatly determined by direct additive genic action, insignificantly affected by maternal phenotype, and with antagonism between the genetic effects. Response to selection for BW would be maximized if based on the parameters obtained by the analysis fitting animal models with direct and maternal effects.
\end{abstract}

Key Words: beef cattle, maternal effects, restricted maximum likelihood, selection

\section{Introdução}

O peso ao nascer (PN) é a primeira informação após o nascimento do animal que indica seu vigor e desenvolvimento pré-natal, fortemente influenciado por fatores ambientais que influenciam a mãe, antes e durante a gestação, e pelo próprio período de gestação. Esta medida é importante para acompa- nhar o desenvolvimento ponderal do animal, além de ser utilizada, eventualmente, para ajustar pesos em idades posteriores (LÔBO, 1996).

Ao mesmo tempo em que baixos PN são indesejados por estarem relacionados ao aumento na taxa de mortalidade na fase pré-desmame, elevados PN tendem a aumentar a ocorrência de distocia nos rebanhos. Como existe correlação genética positiva

\footnotetext{
${ }^{1}$ Parte da Dissertação apresentada à Faculdade de Medicina de Ribeirão Preto pelo primeiro autor para obtenção do título de Mestre em Ciências . Apoio: CNPq e FINEP.

2 Bolsista RHAE-CNPq; Embrapa Arroz e Feijão, Caixa postal 179, 74001970 - Goiânia GO.

${ }^{3}$ Professor Associado do Departamento de Genética da Faculdade de Medicina de Ribeirão Preto- USP. AvBandeirantes, 3900 - $14049-900$-Ribeirão Preto SP; Bolsista CNPq.
} 
de expressiva magnitude entre PN e pesos e ganhos de peso em idades posteriores, principalmente até o desmame, atenção especial deve ser dada a rebanhos em que se faz seleção para peso nesta fase, no sentido de se evitar que o PN médio seja elevado à magnitude tal que possa implicar em desempenho negativo da vaca ao parto e na menor viabilidade de seus produtos, ao nascer.

$\mathrm{O} \mathrm{PN}$, assim como qualquer outra característica, pode ser influenciado por fatores de ambiente, sejam permanentes ou não. Estes efeitos irão agir na vaca, principalmente durante a gestação, aliar-se aos efeitos genéticos e determinar o PN do animal. Dentro de uma mesma raça, diferenças decorrentes de nutrição, condição corporal e idade da vaca, sexo e número de bezerros, ano e estação de nascimento, rebanho, período de gestação, entre outros, são comumente observadas.

Estão disponíveis na literatura estimativas de herdabilidade do PN utilizando-se modelos mistos, assumindo modelo animal, e contemplando também o efeito genético materno que influencia essa característica (Tabela 1). As estimativas obtidas por LÔBO et al. (1994) e REYES et al. (1995) são exemplos, além de serem originárias de uma base de dados que inclui os registros que foram utilizados neste estudo.

O valor do parâmetro pode variar entre estudos, mas de maneira geral a herdabilidade para o efeito direto é de média a alta magnitude, variando de 0,29 (LÔBO et al., 1994) a 0,51 (MEYER et al., 1993; MEYER, 1995), refletindo a expressiva variabilidade genética direta da característica existente em todos os rebanhos e raças estudados. A contribuição materna para a variação dessa característica é relativamente reduzida, em comparação à direta, o que é comumente observado em características de crescimento em bovinos de corte, situando-se, neste caso, entre 0,07 (SWALVE, 1993) e 0,16 (ELER et al., 1996).

Mesmo que a ocorrência de dificuldades ao parto, na maior parte dos plantéis de zebuínos, não atinja índices alarmantes, principalmente porque a média de PN parece ser compatível com o tamanho adulto das vacas, este peso deve ser monitorado, uma vez que existe a tendência de se antecipar a idade ao primeiro parto das primíparas, e não se conhece ao certo o limite entre peso ou tamanho da novilha e peso ao nascer da cria, para um parto sem problemas.

Este trabalho foi conduzido com o objetivo de estudar os aspectos genético-quantitativos envolvidos no peso ao nascer de animais da raça Nelore utilizando diferentes modelos lineares mistos, procurando distinguir entre os efeitos aleatórios que influenciam essa característica.

\section{Material e Métodos}

Para o presente estudo foram utilizadas informações de animais da raça Nelore, criados em oito rebanhos localizados nos Estados de Goiás, Mato Grosso do Sul, Minas Gerais e São Paulo, participantes do Programa de Melhoramento Genético da Raça Nelore (PMGRN).

Foram considerados pesos de bezerros normais, nascidos vivos e oriundos de gestações não gemelares, concebidos por meio da inseminação artificial. Aos rebanhos participantes do PMGRN, é facultativo informar o PN de seus animais. Por motivos práticos, muitos optam por não pesá-los neste evento. Por isso, em algumas fazendas o número de informações de $\mathrm{PN}$ foi reduzido, em relação ao número total de nascimentos.

Todas as informações de genealogia disponíveis foram incluídas com os objetivos de minimizar o vício decorrente de seleção e aumentar a acurácia das estimativas, como conseqüência da conexidade entre as informações de animais (MEYER, 1992). Assim, o arquivo utilizado para implementar a matriz de parentesco, que incluiu também os animais sem registros (animais da população base), foi constituído de 13.684 diferentes animais, dando origem a 13677 pedigrees e a um coeficiente de endogamia de 0,027. $\mathrm{O}$ arquivo de dados para as análises continha informações de 6511 animais com PN conhecido, filhos de 309 touros e 3795 vacas, nascidos entre 1985 e 1996, ao longo de todos os meses do ano.

Foram considerados quatro trimestres de nascimento, que compreenderam os meses de janeiro a março, abril a junho, julho a setembro e outubro a dezembro. A seguir, os animais foram agrupados segundo fazenda, ano e trimestre de nascimento e sexo. Estes procedimentos deram origem aos grupos de contemporâneos (GC), que constituíram um dos efeitos fixos incluídos nos modelos de análise.

As idades das vacas ao parto foram divididas em seis diferentes classes, conforme usualmente feito em estudos e avaliações genéticas pelo PMGRN, constituindo então o segundo efeito fixo. As classes incluíram, separadamente, vacas paridas até os 2 anos, $>2$ a 3 anos, $>3$ a 4 anos, $>4$ a 5 anos, $>5$ a 9 anos e $>9$ anos em diante. Nos casos em que não se conhecia a data de nascimento das vacas, que constituiu uma pequena proporção dos dados, foi atribuída a quinta classe de idade ao parto, por ser a que inclui a média de idade das vacas, ao parto, neste conjunto de dados.

\section{Análise estatística}

O desenvolvimento e a aplicação da teoria do melhoramento genético dependem de modelos mate- 
514 Rev. bras. zootec.

máticos que descrevam, da melhor maneira possível, os sistemas biológicos e suas inter-relações. Com o aumento da capacidade computacional disponível, modelos lineares empregados na análise genética de características e animais têm se tornado mais detalhados. No momento, o modelo linear misto incluindo o animal como efeito genético direto (modelo animal) tem sido aplicado para diversos fins, e sua eficácia tem sido observada na estimação de parâmetros genéticos (MEYER, 1992).

Assim, os componentes de (co)variância foram estimados assumindo-se quatro diferentes modelos, desde o mais simples com um único efeito aleatório, além do erro, até o mais abrangente. Atribuíram-se como causas de variação os efeitos genéticos direto e materno, e, ou, de ambiente permanente, pela inclusão alternada dos efeitos aleatórios pertinentes, além dos efeitos fixos descritos anteriormente. Os modelos podem ser representados sob forma matricial como:

$$
\begin{gathered}
y=X \beta+Z_{1} a+\varepsilon[1] \\
y=X \beta+Z_{1} a+Z_{2} p+\varepsilon \\
y=X \beta+Z_{1} a+Z_{3} m+\varepsilon \\
y=X \beta+Z_{1} a+Z_{2} p+Z_{3} m+\varepsilon
\end{gathered}
$$

em que

$y=$ é o vetor de pesos ao nascimento;

$X=$ é a matriz de incidência dos efeitos fixos;

$\beta=$ é o vetor de efeitos fixos;

$Z_{1}=$ é a matriz de incidência dos efeitos genéticos diretos;

$a=$ é o vetor de efeitos genéticos diretos;

$Z_{2}=$ é a matriz de incidência dos efeitos de ambiente permanente;

$p=$ é o vetor de efeitos de ambiente permanente;

$Z_{3}=$ é a matriz de incidência dos efeitos genéticos maternos;

$m=$ é o vetor de efeitos genéticos maternos;

$e=$ é o vetor de erros aleatórios associados às observações.

Admitiu-se a seguinte distribuição conjunta para os efeitos aleatórios:

$$
\left[\begin{array}{c}
y \\
a \\
m \\
p \\
e
\end{array}\right] \sim \operatorname{NMV}\left\{\left[\begin{array}{c}
X \beta \\
0 \\
0 \\
0 \\
0
\end{array}\right],\left[\begin{array}{ccccc}
V & Z_{1} G & Z_{3} M & Z_{2} P & R \\
G Z_{1}^{\prime} & G & C & 0 & 0 \\
M Z_{3}^{\prime} & C & M & 0 & 0 \\
P Z_{2}^{\prime} & 0 & 0 & P & 0 \\
R & 0 & 0 & 0 & R
\end{array}\right]\right\}
$$

em que

$$
\begin{aligned}
V= & Z_{1} G Z_{1}^{\prime}+Z_{3} M Z_{3}^{\prime}+Z_{1} C Z_{3}^{\prime}+Z_{3} C Z_{1}^{\prime}+Z_{2} P Z_{2}^{\prime}+R \\
& G=\mathrm{A} \sigma_{a}^{2} ; \\
& M=\mathrm{A} \sigma_{m}^{2} ; \\
& C=\mathrm{I} \sigma_{a m} ; \\
& P=\mathrm{I}_{\mathrm{m}} \sigma_{c}^{2} ; \\
& R=\mathrm{I}_{\mathrm{n}} \sigma_{e}^{2} ; \\
& m=\text { número total de vacas; e } \\
n & =\text { número total de observações. }
\end{aligned}
$$

Sendo $\sigma_{a}^{2}$ a variância genética aditiva direta, $\sigma_{m}^{2}$ a variância genética aditiva materna, $\sigma_{a m}$ a covariância entre efeitos genéticos direto e materno, $\sigma_{s}^{2}$ a variância de ambiente permanente materno e $\sigma_{e}$ a variância residual. $\mathrm{G}_{0}, \mathrm{M}_{0}, \mathrm{C}_{0}, \mathrm{P}_{0}, \mathrm{R}_{0}$ são matrizes de variâncias e covariâncias genética direta, materna, de ambiente permanente e residual das duas características, respectivamente. Assume-se que $\mathrm{P}$ ou $\mathrm{P}_{0}$ e $\mathrm{R}$ ou $\mathrm{R}_{0}$ são não correlacionados com os demais efeitos. A é a matriz de coeficientes de parentesco entre os animais e I, uma matriz identidade.

Todos os cálculos foram feitos utilizando-se o sistema MTDFREML (Multiple Trait DerivativeFree Restricted Maximum Likelihood) (BOLDMAN et al., 1995) empregando o procedimento Simplex para localizar o mínimo de $-2 \log \mathrm{L}$, em que log L é o logaritmo da função de verossimilhança restrita (MEYER, 1989). Considerou-se a convergência atingida quando a variância dos parâmetros a serem estimados no Simplex fosse menor que $10-{ }^{9}$, e para assegurar que este valor correspondia ao máximo global de $\log (\mathrm{L})$, e não local, as análises foram repetidas utilizando-se como valores iniciais os obtidos na análise prévia, conforme recomendado por BOLDMAN et al. (1995).

Teste da razão de verossimilhanças

Foi verificada a diferença entre os logaritmos das funções de verossimilhança restrita máximos globais alcançados, com o objetivo de testar a significância da inclusão alternada dos efeitos aleatórios e, conseqüentemente, identificar o modelo capaz de melhor se ajustar aos dados com o menor número possível de parâmetros. Para isso, foram obtidos desvios dos referidos logaritmos, oriundos de modelos que diferiram em apenas um efeito. Estes desvios foram então multiplicados por dois, constituindo o $\lambda$, que por sua vez foi comparado ao valor de qui-quadrado $\left(\chi^{2}\right)$ com um grau de liberdade e à probabilidade mínima de 95\%, conforme descrito a seguir (DOBSON, 1990): 
Tabela 1 - Estimativas de herdabilidade do peso ao nascer segundo grupo genético, método, fonte e país

Table 1 - Estimates of heritability $\left(h^{2}\right)$ for birth weight according to the genetic group, method, source and country

\begin{tabular}{|c|c|c|c|c|c|}
\hline $\begin{array}{l}\text { Grupo genético } \\
\text { Genetic group }\end{array}$ & $\mathrm{h}_{a}^{2}(1)$ & $\mathrm{h}_{m}^{2}$ & $\begin{array}{l}\text { Método }^{2} \\
\text { Method }^{2}\end{array}$ & $\begin{array}{l}\text { Fonte } \\
\text { Source }\end{array}$ & $\begin{array}{c}\text { País } \\
\text { Country }\end{array}$ \\
\hline Nelore & 0,46 & 0,12 & Diversos & ELER etal., 1989a & Brasil \\
\hline Simental & 0,33 & 0,07 & MAM & SWALVE, 1993 & \\
\hline Wokalup & 0,51 & - & MAM & MEYERetal, 1993 & Austrália \\
\hline Hereford & 0,43 & - & MAM & MEYERetal, 1993 & Austrália \\
\hline Diversos & $0,31^{3}$ & $0,14^{(3)}$ & Diversos & KOOTS etal., 1994 & Diversos \\
\hline Nelore & 0,29 & - & MAM & LÔBO et al., 1994 & Brasil \\
\hline Wokalup & 0,51 & - & MAM & MEYER, 1995 & Austrália \\
\hline Hereford & 0,41 & - & MAM & MEYER, 1995 & Austrália \\
\hline Diversos & $0,33^{(3)}$ & $0,12^{3}$ & Diversos & MERCADANTE et al., 1995 & Diversos \\
\hline Nelore & 0,29 & 0,12 & MAM & REYES etal., 1995 & Brasil \\
\hline Nelore & 0,43 & 0,16 & MAM & ELER etal., 1996 & Brasil \\
\hline
\end{tabular}

$1 \mathrm{~h}_{a}^{2}$ : herdabilidade direta; $\mathrm{h}_{m}^{2}:$ herdabilidade materna;

2 MAM: modelo animal multicaráter;

3 Valores ponderados.

$1 \mathrm{~h}_{a}^{2}$ : direct heritability; $\mathrm{h}_{m}^{2}$ : maternal heritability;

2 MAM: multivariate animal model;

3 Weighed values.

$\lambda=2(\log$ likelihood do modelo [b]- $\log$ likelihood do modelo [a])

se $\lambda \geq \chi^{2}{ }_{1,0.95}$ aceita-se o modelo [b]

se $\lambda<\chi^{2}{ }_{1,0.95}$ aceita-se o modelo [a]

em que a é o modelo com $n$ parâmetros e b é o modelo com $n+1$ parâmetros.

\section{Estimativas de parâmetros genéticos}

Além dos componentes de (co)variância e parâmetros genéticos e fenotípicos obtidos em cada uma das análises, cálculos adicionais foram realizados, a fim de se obterem mais informações sobre cada característica e cumprir um dos principais objetivos propostos.

A herdabilidade total $\left(h_{T}^{2}\right)$ proposta por DICKERSON(1947), e que segundo WILLHAM(1972) é a determinante da resposta à seleção para características sujeitas a efeito materno, foi calculada como sendo a regressão do genótipo total do animal em função de seu fenótipo. A expressão de $h_{T}^{2}$ foi calculada como:

$$
h_{T}^{2}=\frac{\sigma_{a}^{2}+0,5 \sigma_{m}^{2}+1,5 \sigma_{a m}}{\sigma_{p}^{2}}
$$

\section{Resultados e Discussão}

A média geral de peso ao nascer de 6511 animais calculada para este conjunto de dados foi de $31 \mathrm{~kg}$, com desvio padrão de $4,1 \mathrm{~kg}$, compatível com boa taxa de sobrevivência dos bezerros e com partos sem distocia. As estimativas resultantes de análises unicaracteres do PN, sob diferentes modelos, estão apresentadas na Tabela 2, e os respectivos valores de $\lambda$, obtidos pela comparação dos modelos, estão apresentados na Tabela 3.

A variância fenotípica diminuiu pouco, porém, continuamente a cada novo modelo estudado, assim como alguns dos parâmetros estimados em sua proporção $\left(h_{a}^{2}, h_{m}^{2}, h_{T}^{2}\right.$ e $\left.\mathrm{c}^{2}\right)$ também diminuíram. As herdabilidades diretas assumiram valores entre $0,37 \mathrm{e}$ 0,20 e as maternas, 0,13 e 0,11 , obtidas a partir dos modelos [1] a [4], e [3] e [4], respectivamente. O efeito de ambiente permanente foi responsável por $9 \mathrm{e}$ $3 \%$ da variação fenotípica observada no $\mathrm{PN}$, conforme observado com os modelos [2] e [4], respectivamente. As herdabilidades totais estimadas a partir de [3] e [4] foram 0,23 e 0,22 , pouco superiores às diretas, não tendo sido tão influenciadas pela covariância negativa entre efeitos genéticos direto e materno.

O modelo [1] forneceu os maiores componentes de variância fenotípica e genética aditiva direta e também de herdabilidade para este efeito, e o resíduo foi o menor entre todos os modelos empregados. A diminuição tanto na variância genética aditiva direta como na sua proporção em relação à fenotípica $\left(h_{a}^{2}\right)$, como conseqüência da inclusão do efeito de ambiente permanente (modelo [2]), foi em torno de $32 \%$, acompanhada de ligeiro aumento no componente residual.

Quando os efeitos genéticos direto e materno foram considerados simultaneamente (modelo [3]), a variância genética direta diminuiu mais do que quando se considerou apenas o efeito de ambiente permanente ([2]). Esta redução foi de $47,5 \%$, contra $33 \%$ do modelo [2], ambos em relação ao [1]. Convém ressal- 
516 Rev. bras. zootec.

Tabela 2 - Estimativas de componentes de (co)variâncias e parâmetros genéticos e fenotípicos para peso ao nascer, obtidos a partir de análises unicaracteres com diferentes modelos

Table 2 - Estimates of (co)variance components and phenotypic and genetic parameters for birth weight, obtained from

Estimativa $^{1}$
uniarivate analysis with different models

\begin{tabular}{lcccc} 
Estimate $^{l}$ & \multicolumn{4}{c}{ Model } \\
\cline { 2 - 5 }$\sigma_{a}^{2}$ & $41]$ & {$[2]$} & {$[3]$} & {$[4]$} \\
$\sigma_{m}^{2}$ & - & -36 & 2,29 & 2,33 \\
$\sigma_{a m}$ & - & - & 1,53 & 1,26 \\
$\sigma_{c}^{2}$ & - & 1,08 & $-0,26$ & $-0,27$ \\
$\sigma_{e}^{2}$ & 7,47 & 7,52 & - & 0,34 \\
$\sigma_{p}^{2}$ & 11,83 & 11,53 & 11,45 & 7,79 \\
$h_{a}^{2}$ & 0,37 & 0,25 & 0,20 & 0,20 \\
$h_{m}^{2}$ & - & - & 0,13 & 0,11 \\
$\mathrm{r}_{\mathrm{am}}$ & - & - & $-0,14$ & $-0,16$ \\
$\mathrm{c}^{2}$ & - & 0,094 & - & 0,029 \\
$\mathrm{e}^{2}$ & 0,63 & 0,65 & 0,69 & 0,68 \\
$h_{T}^{2}$ & 0,37 & 0,25 & 0,23 & 0,22 \\
$\log \mathrm{L}$ & $-42,08$ & $-24,11$ & $-0,97$ & 0 \\
\hline
\end{tabular}

${ }^{1} \sigma_{a}^{2}$ : variância genética aditiva direta; $\sigma_{m}^{2}$ : variância genética aditiva materna; $\sigma_{a m}$ : covariância genética entre efeitos direto e materno; $\sigma_{c}^{2}$ : variância de ambiente permanente; $\sigma_{e}^{2}$ : variância residual; $\sigma_{p}^{2}$ : variância fenotípica; $h_{a}^{2}$ : herdabilidade direta; $h_{m}^{2}$ : herdabilidade materna; $r_{a m}$ : correlação entre efeitos direto $e$ materno; $c^{2}$ : porção da variância fenotípica devida ao ambiente permanente; $\mathrm{e}^{2}$ : porção da variância fenotípica devida ao resíduo; $h_{T}^{2}$ : herdabilidade total; log L: máximo do logaritmo da função de verossimilhança, expresso como desvio do modelo com maior valor.

${ }^{1} \sigma_{a}^{2}$ : direct additive genetic variance; $\sigma_{m}^{2}$ : maternal additive genetic variance; $\boldsymbol{\sigma}_{a m}$ : direct-maternal genetic effects covariance; $\sigma_{c}^{2}$ :variançe due to maternal permanent environmental; $\sigma_{c}^{2}$ : residual variance; $\sigma_{p}^{2}$ : phenotypic variance; $h_{a}^{2}$ : direct heritability; $h_{m}^{2}$ : maternal herdability; $r_{a m}$ : direct-maternal genetic correlation; $c^{2}$ : fraction of phenotypic variance due to maternal permanent environmental; $e^{2}$ fraction of phenotypic variance due to residue; $h_{T}^{2}$ : total heritability ; $\log L$ : $\log$ likelihood, expressed as deviation from model with hightest value.

tar que esta foi a menor estimativa para o referido parâmetro, obtida dentre os quatro modelos estudados, dando origem à menor herdabilidade direta.

Apesar de a variância genética direta obtida a partir de [4] ter sido um pouco superior à obtida em [3], a herdabilidade manteve-se constante. Com a inclusão simultânea dos efeitos maternos, a redução na variância e herdabilidade genética materna já era esperada. A porção da variância fenotípica decorrente do ambiente materno foi de 0,03 , sendo $66,6 \%$ menor que a observada em [2], que por sua vez devia estar acrescida do efeito genético materno. A covariância negativa entre os efeitos genéticos direto
Tabela 3 - Diferenças entre log $L$ obtidas a partir de quatro modelos usados em análises unicaracteres do peso ao nascer

Table 3 - Differences among log $L$ from four models used in univariate analysis for birth weight

\begin{tabular}{lc}
\hline \multicolumn{1}{c}{$\begin{array}{l}\text { Modelo } \\
\text { Model }\end{array}$} & $\lambda$ \\
$\log L[2]-\log L[1]$ & $35,95^{*}$ \\
$\log L[3]-\log L[1]$ & $88,23^{*}$ \\
$\log L[4]-\log L[2]$ & $48,22^{*}$ \\
$\log L[4]-\log L[3]$ & $1,95^{\mathrm{NS}}$ \\
\hline${ }^{*}$ Significativo $(\mathrm{P}<0,05) ;$ NS: não-significativo $(\mathrm{P}>0,05)$.
\end{tabular}

* Significant $(P<0,05) ;$ NS: not significant $(P>.05)$.

e materno, constatada em [3], praticamente não se alterou, assim como a correlação, que foi de $-0,14$ para $-0,16$. A herdabilidade total também sofreu pequena alteração, porém de magnitude desprezível.

O PN, assim como características de crescimento na fase de aleitamento, é influenciado tanto pelo potencial de crescimento do bezerro como pela sua mãe, que fornece o ambiente onde o animal irá se desenvolver. Mesmo assim, também nesta característica, contemplar os efeitos maternos de origem genética e de ambiente, simultaneamente (modelo [4]) não foi significativamente diferente de considerar apenas o efeito genético materno, como feito em [3], talvez porque o ambiente materno permanente realmente seja pouco expressivo, como indica o valor de $\mathrm{c}^{2}$ . Logo, o modelo [3] mostrou-se o mais adequado.

A herdabilidade total obtida, quando se usou o modelo [3], é considerada de média magnitude, indicando que, se for necessário selecionar para o $\mathrm{PN}$, resposta à seleção favorável poderá ser esperada. Em termos práticos, talvez a seleção indireta ou simultânea com outras características positivamente correlacionadas seja mais recomendável.

A máxima verossimilhança supera alguns problemas de estimação de componentes de efeitos maternos, como ocorre nos métodos em que o mesmo animal contribui para diferentes covariâncias (THOMPSON, 1976). Mesmo assim, provavelmente essas estimativas não sejam muito acuradas. Pode ainda haver covariância de ambiente negativa entre mãe e progênie, o que resultaria em estimativa também viciada (MEYER, 1992). Desta forma, deve-se ter cuidado especial ao utilizar estas informações para tomar decisões práticas de seleção, mas procurar sempre considerá-las, uma vez que parece existir relação antagônica com efeito direto.

Os resultados deste estudo constataram antagonismo entre efeitos genéticos diretos e maternos para 
a característica $\mathrm{PN}$, como freqüentemente tem sido observado em estudos nos quais esta relação é estudada. As correlações resultantes de tais covariâncias poderiam reduzir o progresso genético esperado a partir da seleção direta. Se existe correlação genética negativa entre o efeito direto e materno, é porque a mãe fornece para sua progênie bom conjunto de genes para o efeito direto e efeito materno insatisfatório, ou vice-versa, dificultando o melhoramento por seleção baseada no valor fenotípico (WILLHAM, 1972).

Neste contexto, a resposta à seleção para o PN poderá ser maximizada se baseada em parâmetros obtidos em análises com modelos lineares mistos, em que os efeitos genéticos e maternos tenham sido considerados. A herdabilidade total obtida seguindo estes princípios reúne vantagens e tem justificada sua aplicação nos cálculos de resposta esperada à seleção.

Considerando-se a importância do peso ao nascer e suas relações com outras características de interesse em programa de melhoramento genético, sugere-se que os criadores se esforcem para adequar o manejo, a fim de permitir a obtenção desta informação.

\section{Conclusões}

Na população estudada, o peso ao nascer mostrou-se predominantemente influenciado por efeito genético aditivo direto, mas efeitos genético materno e de ambiente permanente também influenciam essa característica. A utilização de um modelo em que não for incluído o efeito de ambiente permanente simultaneamente aos genéticos pode ser interessante, por demandar menos tempo de processamento e memória RAM e se ajustar tão bem aos dados como um modelo completo.

A magnitude do componente de covariância dos efeitos genéticos direto e materno indica antagonismo. Para que a taxa de ganho genético possa ser maximizada, a seleção deve basear-se em parâmetros que o tenham considerado.

As magnitudes dos coeficientes de herdabilidades direta e total indicam que resposta favorável à seleção direta pode ser obtida para esta característica.

\section{Agradecimentos}

Ao CNPq, pela concessão da bolsa de estudo, e à FINEP, pelo apoio financeiro, sem os quais este trabalho não teria sido viabilizado.

Aos criadores participantes do Programa de
Melhoramento Genético da Raça Nelore, pela cessão dos dados.

Ao Analista de Sistemas Luiz Antônio Framartino Bezerra e aos professores Dr. Arcadio de los Reyes, Dr. Elias Nunes Martins e Dr. Henrique Nunes de Oliveira, pelos inúmeros esclarecimentos.

\section{Referências Bibliográficas}

ALVES FILHO, D.C., RESTLE, J. Efeito do grupo genético no peso ao nascimento e ao desmame de terneiros de corte. In: REUNIÃO DA SOCIEDADE BRASILEIRA DE ZOOTECNIA, 34, 1996, Juiz de Fora. Anais... Viçosa: SBZ, 1997. p.202-4.

BOLDMAN, K.G., KRIESE, L.A., VAN VLECK, L.D. et al. A manual for use of MTDFREML. A set of programs to obtains estimates of variances and covariances [DRAFT]. U.S. Department of Agriculture, Agricultural Research Service. 1995.

DICKERSON, G.E. 1947. Composition of hog carcass as influenced by heritable differences in rate and economy of gain. Iowa Agric. Exp. Sta. Bul., 354.

DOBSON, A.J. 1990. An introduction to generalized linear models. Chapman and Hall, Melbourne, $174 \mathrm{p}$.

ELER, J.P., LÔBO, R.B., DUARTE, F.A.M. 1989a. Avaliação dos efeitos genéticos direto e materno em pesos de bovinos da raça Nelore criados no estado de São Paulo. R. Soc. Bras. Zootec., 18(2):112-23.

ELER, J.P., LÔBO, R.B., ROSA, A.N. 1989b. Influência de fatores genéticos e de meio em pesos de bovinos da raça Nelore criados no estado de São Paulo. R. Soc. Bras. Zootec., 18(2):103-11.

ELER, J.P., FERRAZ, J.B.S., SILVA, P.R. Estimação simultânea de parâmetros genéticos para características de importância econômica na raça Nelore, com a utilização de modelos animais. In: REUNIÃO DA SOCIEDADE BRASILEIRA DE ZOOTECNIA, 33, 1996, Fortaleza. Anais... Viçosa: SBZ, 1996. p.99-101.

KOOTS, K.R., GIBSON, J.P., SMITH, C. et al. 1994. Analysis of published genetic parameter estimates for beef production traits.1. Heritability. An. Breed. Abstr., 62(5):309-38.

LÔBO, R.B. Programa de Melhoramento Genético da Raça Nelore. Ribeirão Preto, 1994. 31p.

LÔBO, R.B. 1996. Programa de Melhoramento Genético da Raça Nelore. Ribeirão Preto, 104p.

LÔBO, R.B., REYES, A. de los, FERRAZ, J.B.S. et al. Bivariate animal model analysis of growth weights and scrotal circumference of Nellore cattle in Brazil. In: WORLD CONGRESS ON GENETICS APPLIED TO LIVESTOCK PODUCTION, 5, Guelph, 1994. Proceedings... Guelph, v.17, p.199-201, 1994.

MACHADO, P.F.A., AQUINO, L.H., GONÇALVES, T.M. et al. Influência de fatores de meio sobre características produtivas de animais da raça Nelore. In: REUNIÃO DA SOCIEDADE BRASILEIRA DE ZOOTECNIA, 34, 1996, Juiz de Fora. Anais... Viçosa: SBZ, 1997. p.211-3.

MAGNABOSCO, C. de U., FAMULA, T., LÔBO, R.B. et al. Estimativas de parâmetros genéticos e de ambiente de características de crescimento em bovinos da raça Nelore. In: REUNIÃO DA SOCIEDADE BRASILEIRA DE ZOOTECNIA, 33, 1996, Fortaleza. Anais... Viçosa: SBZ, 1996. p.142-4.

MERCADANTE, M.E.Z., LÔBO, R.B., REYES, A. de los. 1995. Parámetros genéticos para características de crecimiento en 
518 Rev. bras. zootec.

cebuínos de carne. Arch. Lationam. Prod. Anim., 3(1):45-89.

MEYER, K. 1989. Restricted Maximun Likelihood to estimate variance components for animals models with several random effects using a derivative-free algorithm. Gen. Select. Evol., 21:317-40.

MEYER, K. 1992. Variance components due to direct and maternal effects for growth traits of Australian beef cattle. Liv. Prod. Sci., 31:179-204.

MEYER, K. 1995. Estimates of genetic parameters for mature weight of Australian beef cows and its relationship to early growth and skeletal measures. Liv. Prod. Sci., 44:125-37.

MEYER, K., CARRICK, M.J., DONNELLY, B.J.P. 1993. Genetic parameters for growth traits of Australian beef cattle from a multibreed selection experiment. J. Anim. Sci., 71:2614-22.

PENEDO, M.C.T., DUARTE, F.A.M., KOCH, G. 1976. Estudo do período de gestação em gado Nelore. Ciência e Cultura, 28:294 (Suplemento).

REYES, A. de los, LÔBO, R.B., OLIVEIRA, H.N. et al. 1995. Estimación de (co)varianzas y DEPs por modelo animal bicarácter para pesos y perímetro escrotal de ganado Nelore en Brasil. Rev. Arg. Prod. Anim., 15(3/4):926-30.
SAS Institute Inc. SAS User's guide: basics. 5. ed. Cary, NC, SAS Institute, 1985. 1290p.

SWALVE, H.H. 1993. Estimation of direct and maternal (co)variance components for growth traits in Australian Simmental beef cattle. J. Anim. Breed. Genet., 110:241-52.

THOMPSON, R. The estimation of maternal genetic variances. Biometrics, 32:903-17, 1976.

TROVO, J.B.F. Interações genótipo-ambiente em características do crescimento de bovinos Nelore. Ribeirão Preto, 1983. 71 p. Dissertação (Mestrado em Genética) - Faculdade de Medicina de Ribeirão Preto, Universidade de São Paulo, 1983.

WILLHAM. R.L. 1972. The role of maternal effects in animal breeding: III. Biometrical aspects of maternal effects in animals. J. Anim. Sci., 35(6):1288-93.

Recebido em: 18/06/98

Aceito em: 03/12/98 